\title{
Nota preliminar sôbre a variação em orquídeas brasileiras (")
}

\author{
F. G. BRIEGER \\ Secção de Genética \\ Escola S. A. "Luiz de Queiroz" Piracicaba
}

Os estudos sôbre a hereditariedade estão sempre, de uma forma ou outra, ligados às considerações sôbre os mecanismos da evolução, e seria fácil demonstrar, por estudos bibliográficos, que o ponto de partida para os estudos de genética moderna era o desejo de se obterem provas experimentais para a teoria da evolução. Também pode-se constatar que cada descoberta no campo da citogenética foi aproveitada para aprofundar os conhecimentos sôbre os mecanismos da evolução. Supondo que as variações hereditárias observadas em Oenothera eram

(*) Entregue para a publicação em 31-10-1951 
mutações, De Vries formulou a sua teoria sôbre as mutações como mecanismo evolutivo, teoria esta que em princípio está hoje ainda em vigor, apezar do fato que a maioria das chamadas "mutações" em Oenothera são resultados de segregações complexas. Observando o grande número de novas combinações gênicas que aparecem na descendência de híbridos, devido à segregação multifatorial mendeliana, Lotsy formulou a sua hipótese de acôrdo com a qual a hibridação, seguida por segregação e rerombinação mendeliana, seria mais um mecanismo importante da evolução. A descoberta de formas poliploides e aneuploides abriu também caminho para novos estudos e teorias sôbre a marcha da evolução. O ramo mais novo da citogenética moderna, a genética de populações, está abrindo agora novas perspectivas.

A maioria dos estudos feitos sôbre o assunto, em tempos modernos foram realizados na zona temperada do norte, na Europa e na América do Norte, principalmente devido ao fato que a maiori z dos institutos científicos estão localizados nesta zona, quando de outro lado informações sôbre os trópicos e sub-tropicos são mais esparsas.

Devemos admitir que a situação na zona temperada e na zona tropical e sub-tropical é profundamente diferente. $\mathrm{Na}$ primeira é em geral fácil avaliar quais os principais fatores ecológicos aos quais as espécies naturais são adaptadas. Também se conhecêm, pelo menos até certo ponto, as mudanças do clima, causadas tanto pelo avanço como pelo recuo das grandes geleiras que partiram da região ártica e das montanhas. Na zona tropical e sub-tropical ambas as questões tomam uma feição muito majs complexa. Numa floresta tropical temos lado a lado um grande número de "microclimas" e as condições ecológicas, nas quais vivem, aparentemente em conjunto, árvores muito altas, trepadeiras, epífitas e pequenas plantas terrestres, são completamente diferentes. Não houve profundas mudanças do clima em geral desde o terciário. Assim podemos esperar que a marcha da evolução tivesse seguido caminhos diferentes nas duas regiões.

Quando ao reino vegetal, são conhecidas, desde há muito tempo, diferenças nas duas zonas, no que diz respeito ao número de indivíduos por espécie e ao número de espécies que se encontram. na mesma área. $\mathrm{Na}$ zona temperada do norte temos 
em geral por unidade de area, maior número de indivíduos de um menor número de espécies, quando a situação é pelo menos aparentemente, a inversa nos trópicos e sub-trópicos.

Mas antes de procurar uma explicação filogenética ou histórica pará esta diferença, devemos perguntar se se trata de uma regra realmente geral. A obtenção de dadcs estatísticos a respeito é muito difícil. $\mathrm{Na}$ tentativa de uma comparação de associaçõ $\epsilon$ s ecológicas nas regiões climáticas, qual é a unidade de área que devemos tomar em consideração? Para citar um exemplo: para comparar a frequência de espécies e indivíduos num prado de clima temperado, numa floresta da mesma zona e numa floresta pluvial tropical, devemos empregar como unidade da area $1 \mathrm{~m} 2,100 \mathrm{~m} 2$ ou $1.000 \mathrm{~m} 2$ ? Depois vem outra pergunta : o que devemos aceitar como unidade de comparação de ponto de vista da ecologia? Como, por exemplo, podemos definir o que representa uma associação na floresta pluvial tropical; devemos incluir ao mesmo tempo as grandes árvores, o subbosque, a.s plantas herbáceas do chão, as trepadeiras e epífitas, êstes últimos ainda tanto das partes mais sombreadas bem como das coroas das árvores? Finalmente, não temos certeza se os agrupamentos, os quais os sistemáticos denominam de gênero, especie e variedade, representam de fato o mesmo nos trópicos e na zona temperada. Por estas razões, e também porque os dados ainda são esparsos, parece-me difícil aceitar a regra supra mencionada como sendo de ordem geral. Colecionando orquídeas, tivemos, pelo menos até agora, a impressão que a regra não poderia ser aplicada. Encontramos na floresta pluvial da Serra do Mar, numa só árvore, cêrca de 20 espécies de orquideas, cada uma representada por poucos indivíduos, pois não havia espaço para muitos, porém as árvores da visinhança possuiam uma flora idêntica. Tomando como conjunto uma determinada área numa mata, encontram-se em geral relativamente poucas espécies e algumas delas com bastante frequência.

As Orchidaceae apresentam material apropriado para o estudo dos problemas, acima citados, e para comparar o comportamento das espécies de uma família na zona temperada e na zona tropical e sub-tropical. Baseado nas informações, dadas por Pfitzer na sua antiga monografia (1898) que, apezar de ser já um pouco velha, ainda hoje serve para tais compilações, podemos dar o seguinte resumo referente à distribuição geográfica dos 31 grupos de gêneros da familia. 
Grupos com gêneros na zona temperada e em geral nos trópicos de todos os continentes

Grupos com gêneros na América tropical
Grupos com gêneros no mundo velho tropical

DIANDRAE

Cypripedilinae

Apostasiinae

MONADRAE BASITONAE

Ophrydiinae

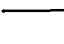

MONANDRAE ACROTONAE ACRANTHAE

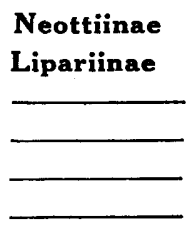

Pleurothallidinae

\section{Laeliinae}

Sobraliinae

Thuniinae

Coelogyninae

Collabinae

Polystachyinae (*)

Podochilinae

Glomeriinae

MONANDRAE ACROTONAE PLEURANTHAE

\begin{tabular}{|c|c|c|c|}
\hline & & Cyrtopodiinae (*) & Phajinae (**) \\
\hline & & Catasetinae & Dendrobiinae \\
\hline & & Lycastinae & Bulbophyllinae (*) \\
\hline & & Gongorinae & Thelasiinae \\
\hline & & Zygopetalinae & Cymbidiinae $(* *)$ \\
\hline & & Steniinae & Thecostelinae \\
\hline & & Maxillariinae (*) & Sarcanthiinae $(* *)$ \\
\hline & & Oncidiinae & \\
\hline & & Dichaeinae & $\longrightarrow$ \\
\hline
\end{tabular}

Nota: (*) Grupos com um gênero de distribuição geral em todo o subtrópico.

(**) Grupos com a maioria dos gêneros no velho mundo, e um ou poucos gêneros na América.

Desta lista podemos tirar as seguintes conclusões :

a) Trata-se, fora de dúvida, de uma família essencialmente tropical e sub-tropical e que iniciou a sua evolução antes da separação cio novo e do velho mundo, pois as sub-famílias não são limitadas a região alguma. 
b) As tribus evoluiram em tempos mais recentes, isto é, depois que a América separou-se dos demais continentes. A existência de algumas poucas exceções não altera em nada esta conclusão, pois em muitas outras famílias de plantas com Araceae etc. podemos observar o mesmo. Em plantas com sementes tão leves e pequenas, como as orquídeas, é realmente de se esperar que às vezes uma ou outra escapasse da área original. Quando isto aconteceu em tempos mais antigos, a forma escapada poderia ainda evoluir e formar hoje um gênero, e quando êste se deu em tempos mais recentes, então encontramos apenas espécies "escapadas".

c) Os grupos que contêm gêneros em todos os continentes são formados por espécies terrestres e que devem possuir, em parte, bastante resistência ao frio, para poder ter penetrado na zona temperada. Em parte pode tratar-se de formas bastante antigas, mas, pelo menos a maioria das espécies da zona temperada do norte, devem ter sofridas alterações na sua distribuição geográfica durante e após o período glacial.

A revisão da sistemática da família, feita por exemplo por Schlechter (1927), consiste num grande aumento do número dos gêneros e dos grupos de gêneros, sendo em ambos os casos o número dobrado. $\mathrm{O}$ aumento do número dos grupos é uma consequência de uma ampla sub-divisão dos grupos de Pfitzer. $\mathrm{O}$ número de gêneros aumentou em parte pelo desdobramento de gêneros antigos, e em parte pela inclusão de material novo. Mas, mesmo adoptando esta classificação, não são alteradas em essência as nossas conclusões.

Assim podemos tirar as seguintes conclusões: Uma vez que os geólogos aceitam a tese que o resfriamento da temperatura no mundo iniciou-se no oligocene e miocene,conduzindo a separação das zonas tropicais do novo e velho mundo, devemos concluir que a diferenciação das sub-famílias iniciou antes e a diferenciação dos grupos de gêneros e dos próprios gêneros depois dêste momento.

Mencionamos acima, que uma dificuldade no estudo de espécies tropicais consiste numa certa dúvida e respeito da separação e identificação das espécies. Esta sempre é dificil em grupos, "exóticos" de ponto de vista dos sistemáticos, que trabalham em geral na zona temperada e assim recebem essencialmente material incompleto e dissecado. Nas orquideas deve- 
mos acrescentar que, devido ao seu valor comercial, sempre existirá uma grande tendência para aumentar exageradamente o númıro de generos e de espécies. Assim parece hoje interessante fazer uma revisão crítica da sistemática, antes de usála para fins científicos modernos.

De fato, a distinção de espécies, nas orquídeas, é às vezes baseada em caracteres bastante superficiais. Temos por exemplo pares de espécies que quase que exclusivamente se destinguem pela época do seu florescimento: Cattleya Loddgesii Ldl. e C. Harrisoniae Batem florescem, a primeira no inverno e a outra no verão, ou C. Warneri Rolfe e C. labiata tip. Ldl. florescem a primeira em outubro-novembro e outra de janeiro em diante, p€lo menos nas condições de Piracicaba. Além dêste fato bem conhecido, há entre as espécies de cada par também diferenças fisiológicas na brotação, pois Loddgesii e Warneri têm um repouso post-floral que parece faltar as outras duas espécies. Em outros casos baseia-se a distinção de espécies sòmente na distribuição da coloração nas flores. Assim encontrei numa só árvore, reunindo numa grande touceira, Maxillaria picta Hook e porphyrostele Ldl., etc., dando esta população mais a impressão de uma segregação genética dentro de uma só espécie, do ques uma mistura de espécie.

A grande variação que o colecionador encontra ou na mesma touceira ou no mesmo bosque, dentro da mata, em espécies como por exemplo C. intermedia Grah, C. Loddgesii Ldl, C. Harrisoniae Batem, L. purpurata Ldl dá a impressão que se trata de segregações mendelianas dentro de populações principalmente reproduzidas por cruzamento livre e referente a gens sem grande importância para a sobrevivência. Mas além disso, não são raros híbridos naturais, intra-específicos, e mesmo intergenéricos.

Para mostrar as dificuldades de forma mais concreta, darei em seguida um resumo dos caracteres e dos agrupamentos dentro de quatro gêneros do grupo dos Laeliinae-Cattleyeae Pfitz ou seja Cattleya, Laelia, Brassavola e Schomburgkia.

A sub-divisão do gênero Cattleya Ldl. (1824) pode ser fàcilmente baseada na forma do labelo, um característico ao qual Pfitzer (1898) atribue importância especial. Combinando a sub-divisão de Pfitzer (1898) e de Cogniaux (1902), podemos distinguir cinco grupos no gênero:

Bicolores Pfitz. sem lobos laterais. labeln

Acklardiae Pfitz. com pequenos lobos laterais na base do 
Guttatae Cogn. com lobos laterais distintos, aproximadamente do comprimento da coluna que êles envolvem.

Intermediae Grah. com lobos laterais bem maiores e uma redução em tamanho do lobo terminal.

Mossiae Pfitz. com lobos laterais grandes e lobo terminal que se distingue apenas por sua coloração.

A Jiferenciação dos órgãos vegetativos não é muito grande nestes cinco sub-gêneros, e podemos distinguir apenas dois ou três tipos diferentes :

Tipo A1 - As hastes verticais são formadas por numerosos internódios compridos e cilíndricos. Esta é a forma mais comum no gênero.

Tipo A2 - As hastes verticais são formadas por poucos internódios, sendo os centrais bastante engrossados de modo que o pseudobulbo inteiro tem uma forma fusiforme: nobilior Reichb. f. e Walkeriana Gardn (do subgen. Acklandiae).

Tipo B - Sòmente o último internódio é grosso e forma o pseudobulbo acentuado. A forma das hastes é claviforme quando os internódios abaixo do último são mais ou menos cilíndricos ou èle é ovoide quando o internódio engrossado nasce quasi diretamente do rizoma.

$O$ tipo A1 termina sempre com duas ou as vezes três fôIhas, o tipo A2 poderá ter uma ou duas folhas e o tipo B sempre possue una só fôlha terminal bem desenvolvida. As demais folhas são sempre reduzidas e formam bracteas ou escamas que cobrem durante algum tempo os internódios.

O tipo A2 é ainda caracterizado pela particularidade que as inflorescências são formadas na ponta de hastes cilíndricas especiais, quando nas demais espécies não há diferença entre hastes vegetativas e florais.

Tomondo em consideração estes caracteres vegetativos, tornase evidente que êles podem servir apenas para subdividir dois dos cinco sub-gêneros. Assim o sub-gênero Acklandiae deve ser dividido f.m dois grupos: Rhizanthemum Cogn, com hastes florais especiais e pseudobulbos do tipo A2, e Acranthemum Cogn. sem hastes florais especiais e pseudobulhos do tipo A1. O subgêneroMossiae poderá ser subdividido em dois grupos: Aurantiaca, do tipo A1 com quatro espécies distribuidas desde o México até o Perú e o Alto Amazonas (aurantiaca Rolfe, Bowrigheana Veitch, luteola Ldl e Skinneri Ldl. e o sub- grupo Labiata, do tipo B com uma distribuição geográfica desde a Columbia e Venezuela até Minas e S. Paulo.

E' interessante notar que o tipo vegetativo A (seja A1 ou A2) ocorre em duas áreas separadas: Os aurantiaca (subgen. 
Mossiae, desde o México até o alto Amazonas e os Bicolores, Acklandiae-Acranthema, Guttatae e Intermediae no Brasil desde Pernambuco até o Rio Grande do Sul, quando o tipo B ocupa a área intermediária da Colombia e Venezuela até Minas e São Paulo.

Deixei fora da discussão a espécie mexicana citrina $L d l$, que conheço apenas de descrições.

A sub-divisão do gênero Laelia $L d l$. (1831) parece muito mais dificil do que o gênero Cattleya, e as tentativas feitas por Pfitzer (1898) Cogniaux (1902) e Schlechter (1917) parecem pouco satisfatorios. Tomando em consideração tanto os caracteres morfológicos, bem como a distribuição geográfica, podemos dividir as espécies provisòriamente em cinco grupos :

1) Crispilabiae com as espécies crispilabia A. Rich., cinnabarina Batem., longipes Reichb f., rupestris Ldl. e caulescensLdl, tôdas da região Mineira, com pseudobulbos mais ou menos cônico-ovoides, mais largos na base do que na ponta; fôlhas bastante grossas; inflorescências com pedúnculos bastante longos quando comparado com o tamanho dos pseudobulhos, compostos de vários internódios; labelo claramente dividido em três lobos. Trata-se de um grupo bastante distinto e que parece ser uma unidade natural. $\mathrm{Na}$ classificação dada acima poderiamos incluir c tjpo dos crispilabiae como tipo A 3. Também devemos incluir aqui, como tipo com adaptação especial, a $L$. harpohylla Reich.j. f., (Minas, Espírito Santo, etc.), na qual todos os internódios são finos e cilíndricos, sendo porém o último muito mais comprido do que os demais.

2) Regnelliae com duas espécies (Regnellii Barb. Rodr. e Lundii Reich f., a primeira do Estado de São Paulo e a outra de Minas) com pseudobulbos bem pequenos, ovoides, terminando em duas folhas finas e bastante longas, inflorescências mais ou menos do comprimento das folhas, labelo trilobado.

3) Purpuratae com cêrca de dez espécies brasileiras (Baía até Rio Grande do Sul), com hastes iguais ao tipo B descrito para as Cattleyas, variando as dimensões nas diferentes espécies. L. pumila Reichb. f. parece, à primeira vista, não pertencer neste grupo, pois as hastes são cilíndricas, mas mesmo nesta espécie o último internódio é muito mais comprido do que os demais, alem de ser um pouco mais grosso, formando assim o pseudobulbo propriamente dito. Tôdas as espécies do grupo têm uma única folha grande na ponta do pseudobulbo. As inflorescências têm em geral pedúnculos relativamente curtos. Os labelos são às vezes mais, as vezes menos, divididos em três lobos. 
A êstes três grupos de espécies exclusivamente brasileiros, temos que acrescentar mais dois grupos de espécies que ocorrem do México até Panamá.

5) Speciosae com cêrca de seis espécies do México, Guatemala e Fanamá, e que possuem psudobulbos do mesmo tipo como os purpuratae ou as labiatae no gênero Cattleya, terminando com uma e raras vezes duas folhas. As inflorescências, inclusive $\mathrm{c}$ seu pedúnculo, são em geral bem mais compridas do que os pseudobulbos, exceto em Johniana Schlecht. Nos labelos encontramos uma divisão em três lobos.

6) Superbiens, com as espécies superbiens Ldl, furfuracea $L d l$ e autumnalis $L d l$, de México e Guatemala, nas quais vários internódios tomam parte na formação dos pseudobulbos. Em superbiens os internódios são grossos e ao mesmo tempo longos e cilíndricos, dando o aspecto de uma Cattleya do tipo A1 bastante grossa, quando os pseudojulbos de autumnalis e furfuracea são ovoides e assim parecem variantes mais grossas, do tipo A2 de Cattleya. As inflorescências, inclusive os pedúnculos são bastante longos, e os labelos divididos em três lobos.

Ccmo terceiro gênero citarei o gênero Brassavola $R$. Br. (1813) ne qual podemos distinguir quatro grupos, mais ou menos naturais :

1) Perrinii, com hastes e folhas terminais cilíndricas e labelo com margem completamente lisa. Seis das espécies encontram-se na área brasileira, de Minas até Sta. Catarina, quando outra espécie, acaulis $R$. Br., segundo Schlechter (1927) é encontrada na Guatemala.

2) Martianae, (com uma distribuição desde a bacia do Amazonas até a Venezuela, as Guianas e algumas ilhas das Antilhas), tem o mesmo porte como o grupo anterior, mas a margem do labelo é dentado, em vez de liso, ou mesmo levemente fimbriado.

3) As Nodosae, conforme as descrições de Schlechter (1927) têm um porte não muito diferente das formas anteriores, mas as folhas, $\epsilon m$ cortes transversal, têm a forma de um hemisfério. A margem do labelo é lisa. As três espécies do grupo são encontradas na área do mar das Caraibas.

4) As Digbyanae, com as duas espécies Digbyana $L d l$ e glauca $L d \mathfrak{d}$, ambas da área do México e América Central (Guatemala, Honduras, segundo Schlechter) têm pseudobulbos que lembram o tipo das labiatae no gênero Cattleya e das purpuratae das Laelias. 
Finalmente teremos que referir-nos ao gênero Schomburgkia Ldl. (1838). Conhecendo pessoalmente uma só espécie, tenho que basear-me principalmente nas referências de Pfitzer (1908), Cogniaux (1902) e Schlechter (1927). A área ocupada extende-se desde o México, no Norte, até São Paulo, no Sul, sem que existisse uma área com maior densidade de espécies. Pela morfologia podemos distinguir dois grupos:

1) Crispae que repetem o tipo $B$ das labiatae no gênero Cattleya e das purpuratae do gênero Laelia, tendo porém sempre duas folhas terminais e um pedúnculo bastante comprido da inflorescência.

2) Tibicines, com pseudobulbos longos e finos, fusiformes em vez de claviformes. O labelo é às vezes mais, às vezes menos pronunciadamente trilobado, sendo o lobo frontal sempre o maior.

Depois da descrição dos grupos dos quatros gêneros, passaremos ảar a caracterização dos gêneros mesmos. Deixei êste ponto para o fim, para antes demonstrar que deve haver bastante afinidade entre êles. De fato, as diferenças genéricas não nos parecem ser muito profundas e às vezes até obscuras.

A única diferença entre Laelia e Cattleya consiste no fato que tôdas as espécies incluidas no primeiro têm oito polinias e aqueles do outro gênero apenas quatro polinias por flor. Apezar que todos os sistemáticos, e os orquidófilos aceitaram desde há mais de cem anos a separação dos dois gêneros, feitos por Lindley em 1821 e 1831, temos que levantar a questão, se neste caso a tiferença do número de polinias não poderia ser apenas um característico secundário na evolução.

No que diz respeito aos três primeiros grupos de espécies do gênero Erassavola, podemos constatar que a sua aparência é bastante típica, e não se repete em nenhum dos demais gêneros das Laellinae, justificando o seu destaque em gênero. Porém as Digbyanue dificilmente podem ser separadas do gênero Laelia, e Pfitzer de fato incluiu-se neste último gênero. Porém todas as espécies do genero Brassavola tem um caraterístico em comum: o pescoço longo dos pontos.

O gênero Schomburgkia é caracterizado pelo fato que as sépalas \& pétalas são sempre onduladas e que a base do labelo está um pouco concrescida com as margens da coluna. Todavia, todos os autores afirmam que os gêneros Laelia e Schomburgkia, ambos com oito polínias, estão bastante próximos.

Este breve resumo demonstra que todos os quatro gêneros formam um conjunto natural. Êste fato é ainda mais claramente demonstrado pelo fato que êles formam fàcilmente híbridos 
quando experimentalmente cruzados, fato êste bem conhecido dos orquidófilos. Mais importante ainda é que pelo menos espécies dos gêneros Laelia e Cattleya também formam híbridos naturais, como por exemplo $L$. purpurata $L d l$ com C. intermedia Grah (LC. Schillerianae Rolfe) e com guttata Ldl (LC. elegans Reichb. f.) ou L. lobata Veitch com C. intermedia Grah (LC Amanda Reichb. f.) ou ainda L. tenebrosa Rolfe com C. Warneri Mioore. (LC. Gottoiana Rolfe).

Assim parece interessante estudar em detalhe o comportamento dos híbridos, e conheço de experiência própria apenas alguns híbridos em geração $\mathrm{F} 1$. De modo geral pode-se dizer que os híbridos, em F1, são intermediários, às vezes sendo um ou outro pai predominante, em determinados característicos. No que diz respeito ao comportamento dos caractéres morfológicos e vegetativos podemos dizer o seguinte: No cruzamento $C$. Loddegesii Ldl x Walkeriana Gardn. (tipo A1 x A2) encontramos pseudobulbos mais ou menos cilíndricos, sendo os últimos internódios um pouco mais compridos e grossos do que os demais. E' interessante notar que há semelhança com os pseudobulbos de L. pumila Reichb. f., que classificamos como um tipo especial do tipo B. Nos cruzamentos de espécies do tipo A1 com outras de tipo $B$, obtemos em $F 1$ sempre uma variante do tipo B, sendo o último internódio sempre mais comprido e grosso do que os demais, sem atingir porém a grossura normal do tipo B, como por exemplo nos cruzamentos de C. intermedia Grah ou Joddegesii Ldl com várias formas de C. labiata $L d l$ ou com L. purpurata Ldl.

Assim podemos tirar a conclusão que o agrupamento das espécies será bastante diferente se empregamos como característico orítico ou a estrutura vegetativa, por exemplo, os diferentes tipos de porte (A1, A2, B, etc.), ou a estrutura floral, por exemplo, o número de polínias, a divisão do labelo, etc. Seja qual for o critério preferido e ao qual atribuimos mais importância na formação filogenética das espécies e gêneros, sempre temos que admitir que há muita variação paralela nos caracteres aos quais se atribui menos importância filogenética. Esta variação paralela poderia ter uma explicação na forma seguinte: Uma vez que as espécies dos diferentes gêneros são descendentes de formas ancestrais comuns, elas ainda contém gens de constituição semelhante e que por isso tendem a produzir mutações com efeitos fenotípicos idêntiticos. Porem esta explicação parece pouco provável, se lembramos que se trata de caracteres genotípicos muito complexos e que dificilmente podem depender de apenas poucas mu- 
tações gêricas. Assim resta outra explicação: a hibridização introgressiva ("introgressive hybridization" de Edgar Anderson). E' um fato conhecido e já mencionado acima, que cruzamentos interspecíficos e intergenéricos são fàcilmente obtidos e ocorrem na natureza. Sendo êstes híbridos em geral férteis, devemos esperar uma forte segregação mendeliana nos seus descendentes, bem como, em retrocruzamentos, a transferência de gens de uma espécie ou de um gênero para outro. Ambas as explicações preliminares são passiveis de comprovação, peìo menos parcialmente, em experimentos genéticos, de modo que não podemos e nem devemos ainda tentar chegar a uma decisão final.

Finalmente quero fazer referência a um problema filogenético muito importante. $\mathbf{E}$ uma tese, já bastante antiga e hoje de novo fortemente defendida por cientistas de destaque como Dobzhansky, Mayr e outros, que a separação filogenética em espécies, isto é o processo de especiação, exige antes de mais nada a existência de um mecanismo eficiente de isolamento sexual que assim permite uma evolução divergente e em separado. Os mecanismos de isolamento são bastante numerosos, como se pode ver nas classificações dadas pelos autores, por exemplo, na classificação de Dobzhansky modificada por Brieger. A existência de híbridos simpátricos entre gêneros e espécies de orquídeas de um lado, o fato que ao mesmo tempo existem e são mais frequêntes os indivíduos das espécies puras, demonstra que devem existir, mesmo de forma incompleta, mecanismos de isolamento reprodutivo.

Em resumo, depois de estudos ainda preliminares podemos já fccalizar três problemas de interesse fundamental, que podem ser estudados nas orquídeas brasileiras: a) Apesar da regra sôbre a proporção entre o número de indivíduos e o número de espécies na mesma área, as orquídeas parecem aproximar-se mais a proporção que prevalece nas zonas temperadas do que aquela dos trópicos, apesar de se tratar de um grupo de plantas $\mathrm{d} \varepsilon$ origem tropical.

b) Deve ser achada uma explicação para a ampla variação paralela que existe em diferentes gêneros, e que pode ser um resultado de uma híbridação introgressiva ou de mutação paralela.

c) Apesar da existência de híbridos simpátricos, tanto intergenéricos como inter-específicos, devem existir mecanismos de isolamento reprodutivo, mesmo incompletos, e que garantem a preservação das espécies puras. 


\section{SUMMARY}

Preliminary studies on brazilian orchid species show that the existing material offers the possibility for studying the following general questions :

a) In spite of the rule about the relative proportion between numbers of individuals per species and number of species per unit area in tropical and in temperate zones, the orchid genera seem to follow more closely the rule of temperate zone species, and this in spite of the fact that the family is undoubtedly of tropical origin.

b) The large amont of paralell variation existing between different genera recquires a detailed explanation, and extensive introgressive hybridization is mentioned as one possibility.

c) The existence of natural sympatric hybrids, both intergeneric and interspecific, and the simultaneous existence of the species in the pure form, show that mechanismes of reproductive isolation must exist, though of an incomplete nature.

\section{BIBLIOGRAFIA}

COGNIAUX, A., 1902 - Orchidaceae. Laeliinae Martius, Flora Brasiliensis Vol. III, 5.

PFITZER, E., 1889 - Orchidaceae Engler--Prantl, Die natuerlichen Pflanzen-familien, II, 6.

SCHLECHTER, R. 1917 - Die Einteilung der Gattung Laelia und die geographische Verbreitung ihrer Gruppen. Orchis 9: 87-96.

SCHLECHTER, R., 1927 - Die Orchideen. Berlin, Parey. 
